\title{
Preview of ZEUS Results
}

\author{
Massimo Corradi * \\ INFN Bologna \\ Via Irnerio 46, I-40126 Bologna - Italy
}

Recent results from the ZEUS experiment are reviewed. They provide new constraints on parton densities, limits on new physics and precise tests of QCD.

\section{Introduction}

The HERA running at high energy $(\sqrt{s}=$ $300-318 \mathrm{GeV}$ ) ended on $20^{\text {th }}$ March. At the time of this conference, HERA was running with reduced proton energy $\left(E_{e}=27.5 \mathrm{GeV}\right.$, $E_{p}=460 \mathrm{GeV}, \sqrt{s}=225 \mathrm{GeV}$ ) with the main purpose of providing a second energy point to measure the longitudinal structure function $F_{L}$ (see Section 8). The full luminosity collected by ZEUS in high-energy runs and available for

\begin{tabular}{|l|r|r|}
\hline Period & $e^{+} p$ & $e^{-} p$ \\
\hline HERA-I & $115 \mathrm{pb}^{-1}$ & $17 \mathrm{pb}^{-1}$ \\
\hline HERA-II (pol.) & $182 \mathrm{pb}^{-1}$ & $190 \mathrm{pb}^{-1}$ \\
\hline Total & $297 \mathrm{pb}^{-1}$ & $207 \mathrm{pb}^{-1}$ \\
\hline
\end{tabular}

Table 1: Useful luminosity collected by ZEUS at $\sqrt{ } s=300-318 \mathrm{GeV}^{2}$ physics analysis is about $0.5 \mathrm{fb}^{-1}$. The breakdown according to the lepton-beam charge and to the lepton beam being unpolarised (HERA-I) or polarised (HERA-II) is given in Table 1.

Many new physics results have been released by the ZEUS collaboration since DIS2006. They consist of the final publications of HERA-I analyses and preliminary results from the large $e^{-} p$ data sample collected in the 2005-2006 period, with some analysis of the full data sample including the last $e^{+} p$ run from 2006-2007. A brief overview of some of the latest results is given here.
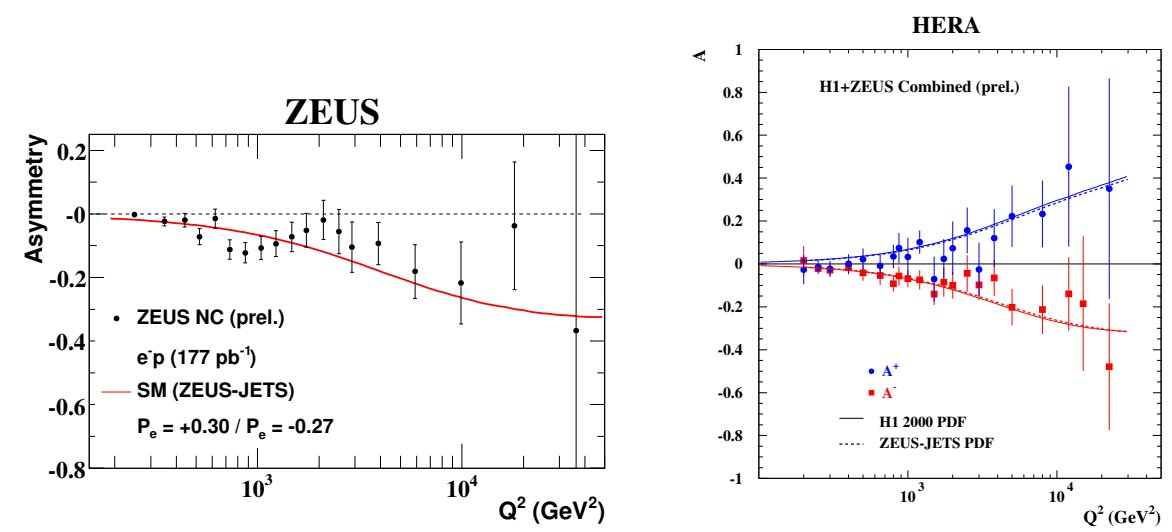

Figure 1: Polarisation asymmetry from ZEUS $e^{-} p$ DIS (left) and from combined H1 and ZEUS $e^{+} p\left(A^{+}\right)$and $e^{-} p\left(A^{-}\right)$data (right).

*also Univ. Hamburg I, Von Humboldt Fellow. 
ZEUS

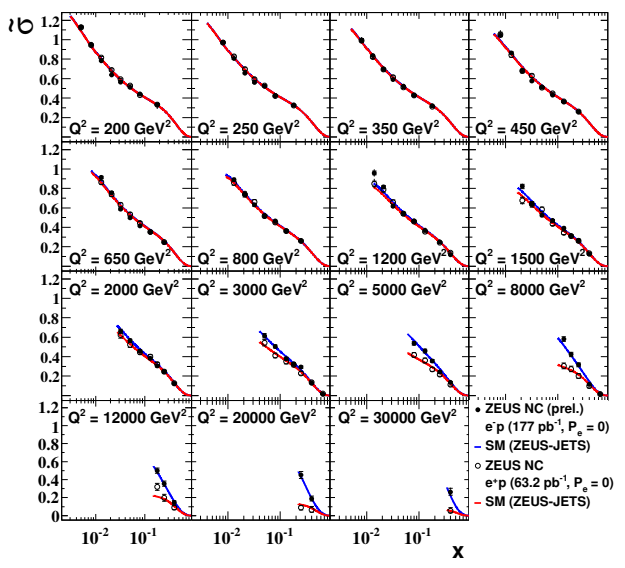

ZEUS

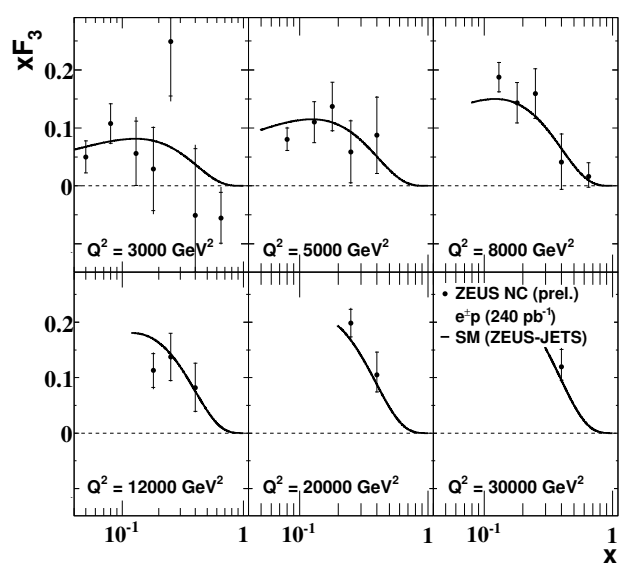

Figure 2: Reduced cross-section for unpolarised $e^{-} p$ and $e^{+} p$ DIS (left) and the structure function $F_{3}$ (right).

\section{Neutral Current DIS at high $Q^{2}$}

The polarised $e^{-} p$ data sample, which consists of $72 \mathrm{pb}^{-1}$ of collisions with right-handed polarised electrons (average polarisation $P_{R}=+0.30$ ) and $105 \mathrm{pb}^{-1}$ with left-handed polarisation $\left(P_{L}=-0.27\right)$, was used to investigate parity violation effects in neutral current (NC) DIS at large $Q^{2}$.

The Standard Model (SM) predicts a dependence of the NC DIS cross-section on the lepton beam polarisation, arising mainly from the $\gamma-Z^{0}$ interference term. The polarisation asymmetry, defined from the cross sections of the right- and left-handed samples $\sigma_{R, L}$ as

$$
A=\frac{2}{P_{R}-P_{L}} \frac{\sigma_{R}-\sigma_{L}}{\sigma_{R}+\sigma_{L}}
$$

is expected to be suppressed by the $Z^{0}$ propagator and proportional to the quark vector coupling to the $Z^{0}, v_{q}$. Figure 1 (left) shows the polarisation asymmetry measured with the ZEUS $e^{-} p$ data as a function of $Q^{2}$ compared the the SM prediction using the ZEUSJETS parton density functions (PDFs) [2]. Good agreement is observed. To increase the statistical significance of this measurement, data from the $\mathrm{H} 1$ and ZEUS collaborations have been combined. The combined polarisation asymmetries for the $e^{+} p$ data collected in 20032004 and part of $e^{-} p$ data, for a total luminosity of $478 \mathrm{pb}^{-1}$, are shown in Fig. 1(right). The asymmetry for $e^{+} p$ has opposite sign to that for $e^{-} p$, in agreement with the SM [3]. The presence of parity violation in high $-Q^{2}$ NC DIS is well established from these data.

The two samples with opposite polarisation have been combined to measure the NC cross section for unpolarised $e^{-} p$ DIS. Figure 2 (left) shows the reduced cross section $\tilde{\sigma}=$ $\frac{d \sigma}{d x d Q^{2}} \frac{x Q^{4}}{2 \pi \alpha^{2} Y_{+}}$, where $Y_{ \pm}=1 \pm\left(1-y^{2}\right)$, compared to $e^{+} p$ data from HERA-I and to SM predictions. At large $Q^{2}$ the cross section for $e^{-} p$ is larger than for $e^{+} p$, again due to the 
effect of the $\gamma-Z^{0}$ interference, and is proportional to the structure function $F_{3}$ :

$$
\tilde{\sigma}\left(e^{-} p\right)-\tilde{\sigma}\left(e^{+} p\right)=2 \frac{Y^{-}}{Y^{+}} x F_{3} \sim 2 \frac{Y^{-}}{Y^{+}} \sum(q-\bar{q}) 2 e_{q} a_{q} a_{e} \chi_{Z},
$$

where $\chi_{Z}=\frac{Q^{2}}{Q^{2}+M_{Z}^{2}} \frac{1}{\sin ^{2} 2 \theta_{W}}, e_{q}$ is the quark electric charge, and $a_{q}$ and $a_{e}$ are the axial couplings to the $Z^{0}$ of quarks and electrons, respectively.

The measurement of $x F_{3}$ from the lepton-charge dependence provides a direct probe of the valence-quark distributions using a pure proton target. The measured structure function $F_{3}$ is presented in Fig. 2 (right). It is in good agreement with the SM prediction based on the ZEUS-JETS PDFs.

\section{Search for new physics}

NC DIS data at high $Q^{2}$ can be used to search for new physics that could contribute to the $e q \rightarrow e q$ amplitude when large scales are involved. In an analysis of $274 \mathrm{pb}^{-1}$ of HERA-I and polarised HERA-II data, no significant deviation from the SM was found, providing competitive limits on contact interactions, large extra dimensions and quark substructure [4]. As an example, Fig. 3 shows the ratio of the number of events observed and expected by SM using the CTEQ5D PDFs, as a function of $Q^{2}$ for

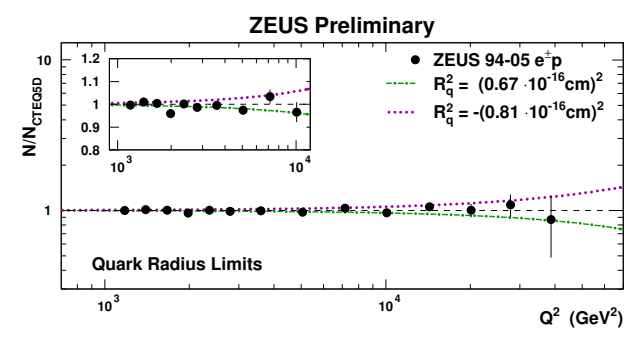

Figure 3: Quark radius the whole sample. A finite quark size would show up to first order as a deviation of the SM cross section of the form

$$
\frac{d \sigma}{d Q^{2}}=\left(\frac{d \sigma}{d Q^{2}}\right)_{\mathrm{SM}}\left(1-\frac{1}{6} R_{q}^{2} Q^{2}\right)^{2}
$$
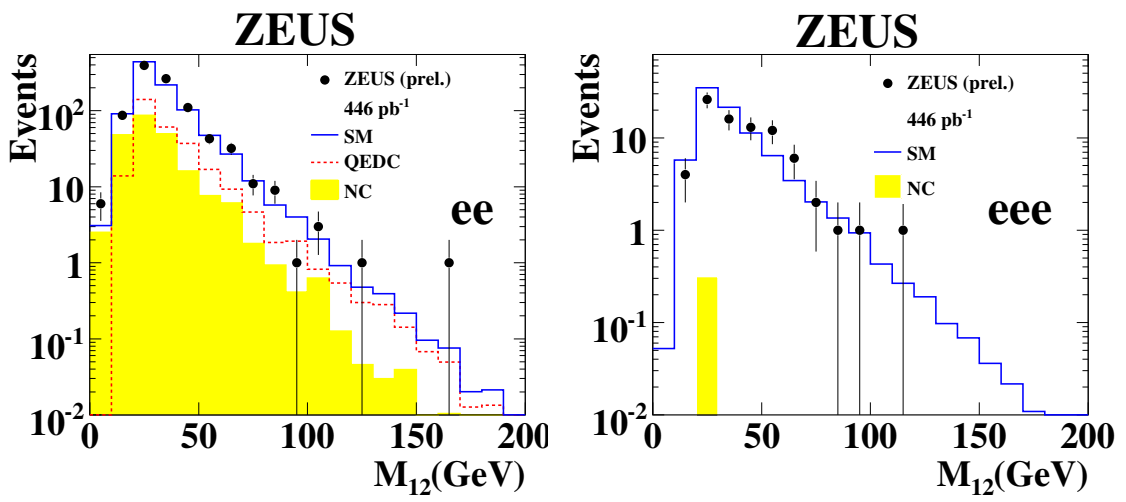

Figure 4: Multielectron mass distributions 
where $R_{q}^{2}$ is the RMS radius of the electroweak charge distribution of the quark. The dotdashed line corresponds to the upper limit $R_{q}<0.67 \times 10^{-3} \mathrm{fm}$.

New physics could also show up as events with uncommon topologies in the SM, such as multileptons or events with leptons and missing momentum. Figure 4 shows the mass distribution for events with two electrons with $p_{T}^{e}>10,5 \mathrm{GeV}$ and for events with a third additional electron with energy $E_{e}>10 \mathrm{GeV}$ ( $5 \mathrm{GeV}$ if rear), updated to almost all the collected luminosity. The data agree with the SM expectation which is dominated by the $\gamma^{(*)} \gamma \rightarrow e^{+} e^{-}$process, with some background in the two-electron sample at high mass from QED Compton events in which the photon was misidentified as an electron [5].

The search for events with an high- $p_{T}$ isolated lepton and large missing momentum was extended to $432 \mathrm{pb}^{-1}$ both in the electron and muon channel. The data are in agreement with the SM prediction which is dominated by $W$ boson production [6]. The H1 collaboration has been reporting a possible excess of events with isolated lepton and missing momentum plus a hadronic system with transverse momentum $p_{T}^{X}>25 \mathrm{GeV}$ in $e^{-} p$ collisions. In the ZEUS case, 7 events $(5 e+2 \mu)$ of this kind have been found in $e^{-} p$ data, in agreement with the SM expectation of 6 events. Similarly, the 4 events $(1 e+3 \mu)$ found in the $e^{+} p$ sample agree with the 6 expected by the SM.

\section{Jet physics}

Jet production provides a precise means to test QCD and to probe the gluon density in the proton, $g\left(x, Q^{2}\right)$. In particular, the inclusive production of high- $E_{T}$ jets in DIS, measured with the $k_{T}$ algorithm in the Breit frame, can be calculated with small theoretical uncertainties in next-to-leading order (NLO) QCD. The analysis of inclusive jets in HERA-I data has been completed recently [7]. Figure 5(left) shows the dependence of the jet cross section on the radius parameter $R$ of the $k_{T}$ algorithm for $Q^{2}>125 \mathrm{GeV}^{2}$. For $R=1$, the data have an uncertainty of $\sim 5 \%$ dominated by the calorimeter energy scale, while the theoretical uncertainty is about $10 \%$. Both uncertainties decrease with $Q^{2}$, to $\sim 3.5 \%$ and $\sim 5 \%$, respectively, for $Q^{2}>500 \mathrm{GeV}^{2}$. The NLO calculation can reproduce the data well for $R$ between 0.5 and 1 , provided that hadronisation correction, which become large at small $R$, are applied. Comparing the rate of jets with $E_{T}^{\text {Breit }}>8 \mathrm{GeV}$ in events with $Q^{2}>500 \mathrm{GeV}^{2}$ to NLO QCD predictions, one of the most precise measurement of the strong coupling constant $\alpha_{S}$ has been obtained:

$$
\alpha_{S}\left(M_{Z}\right)=0.1207 \pm 0.0014 \text { (stat.) } \pm 0.0035 \text { (syst.) } \pm 0.0023 \text { (theo.). }
$$

While the measurement of inclusive jets has the smallest experimental and theoretical uncertainties, the study of dijets provides a more direct probe of the gluon density since the two-jet kinematics allows the reconstruction of the parton momentum fraction $\xi$ and to select regions dominated by the boson-gluon fusion process. The analysis of events with two jets with $E_{T}^{\text {Breit }}>12,8 \mathrm{GeV}$ has been extended to part of the HERA-II data for a total of $209 \mathrm{pb}^{-1}$ [8]. The agreement with NLO QCD, shown in Fig. 5(right), is very good. At large $\xi$ and large $Q^{2}$, where the uncertainties are still dominated by statistics, the analysis of dijets can add a significant constraint to $g(x)$.

High- $E_{T}$ jets in NC DIS appear to be well understood. It is anyway important to check that QCD predictions for high- $E_{T}$ jets also hold in other processes. Charged current $(\mathrm{CC})$ DIS provides an alternative sample in which jet production can be studied. Inclusive, two- 

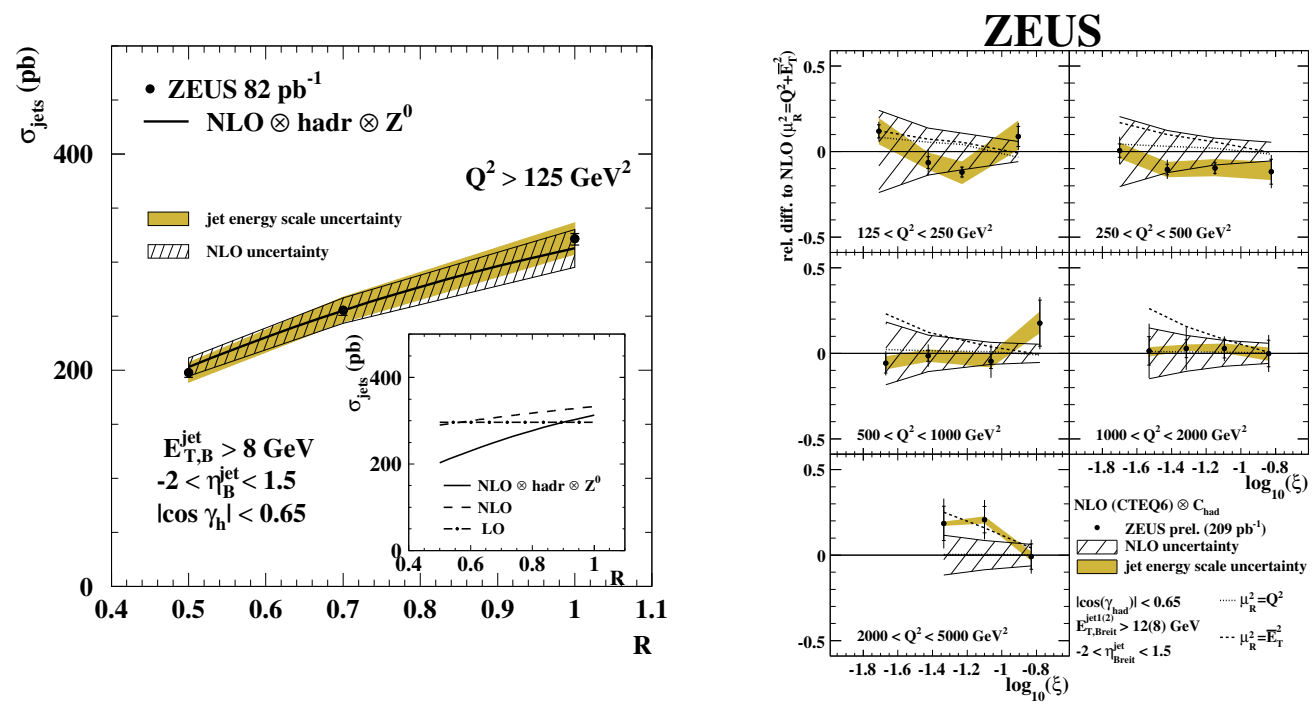

Figure 5: Left: inclusive jet cross section in the Breit frame in NC DIS for different values of the jet radius parameter $R$ of the $k_{T}$ algorithm. Right: data/theory ratio for dijets in DIS.
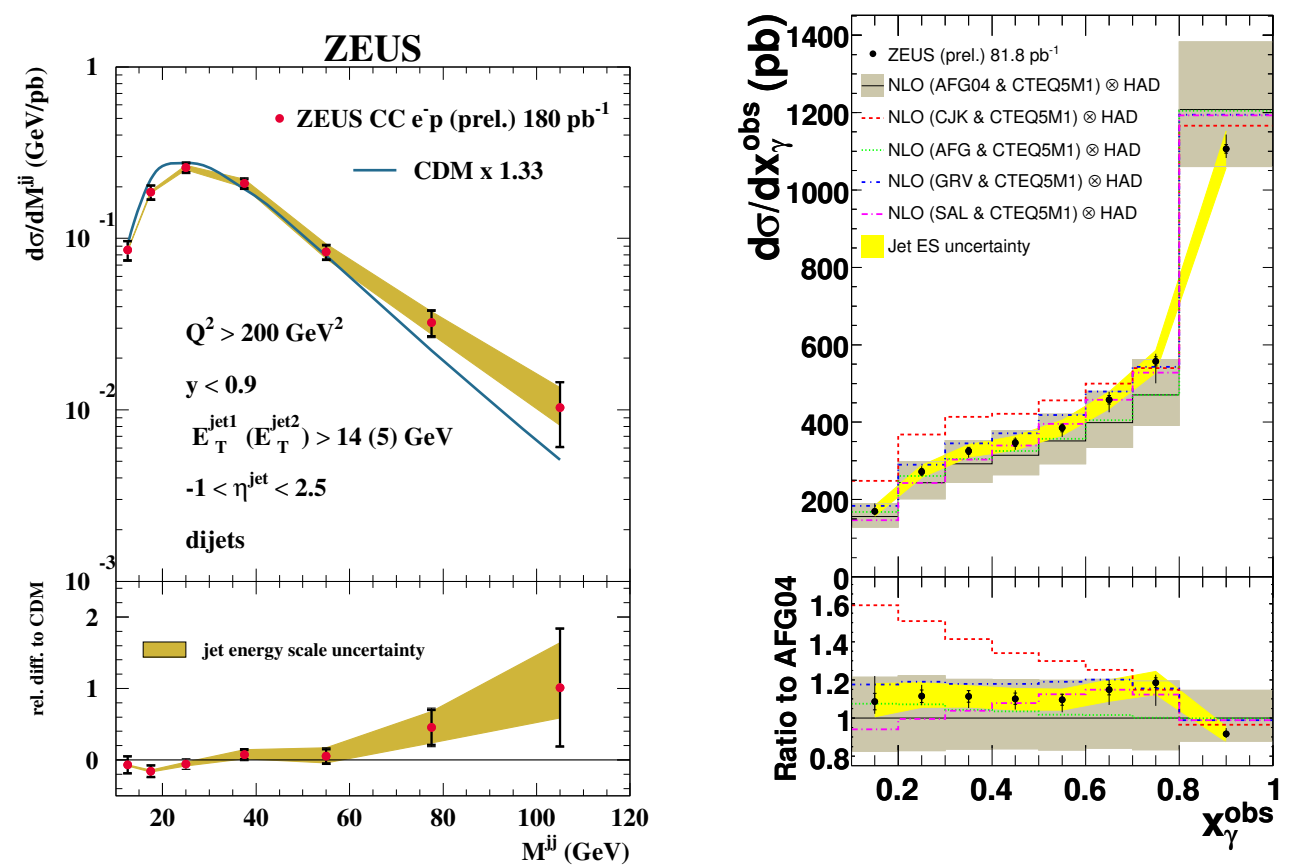

Figure 6: Left: two-jet mass distribution in CC DIS. Right: dijet cross-section in photoproduction as a function of $x_{\gamma}^{\text {obs }}$ 
and three-jet cross sections have been studied for the first time in a large $e^{-} p$ CC DIS sample of $209 \mathrm{pb}^{-1}$. The cross section for two jets in the laboratory frame is shown in Fig. 6(left) as a function of the dijet mass. The agreement with the ARIADnE Monte Carlo is satisfactory [9]. A better insight will be obtained when a comparison with NLO QCD will be available.

High- $E_{T}$ dijets in photoproduction (i.e. $Q^{2}<$ $1 \mathrm{GeV}^{2}$ ) are another sensitive tool to constrain the gluon density in the proton at large $x$. Resolvedphoton events, in which the photon behaves as a source of partons, provide also sensitivity to the gluon density inside the photon. Cross sections for events with two jets with $E_{T}>20,15 \mathrm{GeV}$ and with pseudorapidity $\eta<3$ for the most forward and $-1<\eta<2.5$ for the most backward jet, have been measured and compared to different NLO QCD predictions based on different parton densities. Figure 6(right) shows the cross section as a function of $x_{\gamma}^{\text {obs }}$, the fraction of photon momentum taken by the two-jet system in the proton rest frame, compared to NLO QCD predictions computed with different photon PDFs. At large $x_{\gamma}^{\text {obs }}$, all the curves are in good agreement with the data. In the low $x_{\gamma}^{\text {obs }}$ region, dominated by resolved-photon processes, the theoretical curves can differ by up to a factor two, reflecting the poor knowledge of $g(x)$ of the photon. The dijet data have sufficient precision to distinguish among different parametrisations [10].

The jet analyses described so far aim at a comparison with precise QCD predictions and therfore

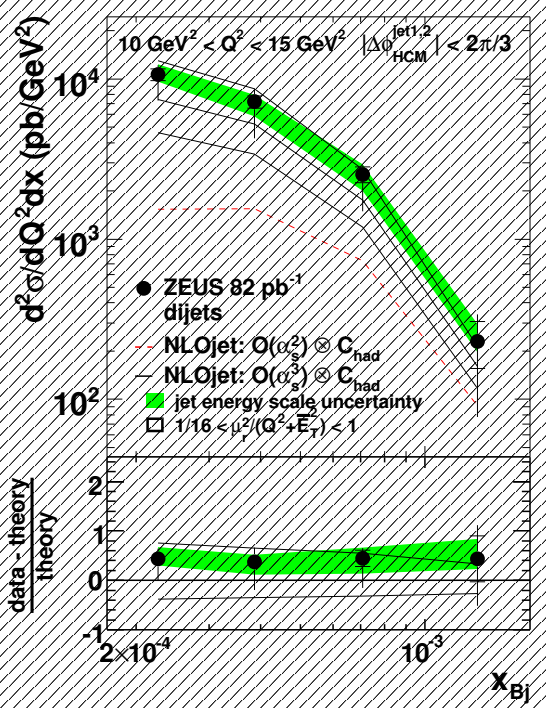

Figure 7: Cross section for unbalanced jets (azimuthal separation $\Delta \Phi<2 \pi / 3$ ) in DIS, compared to $\mathcal{O}\left(\alpha_{S}^{3}\right)$ and $\mathcal{O}\left(\alpha_{S}^{2}\right)$ QCD calculations. focus on the high- $E_{T}$ region where perturbative QCD is expected to be more reliable. It is also interesting to look at regions of the phase space where the standard QCD predictions based on NLO matrix elements and collinear factorisation are expected to break down. This is the case of the low- $x$ regime, where large logarithms of $1 / x$ could spoil standard DGLAP evolution and where saturation effects may show up. These effects could be too small to produce a significant deviation from DGLAP evolution in inclusive DIS data but could be revealed by particular jet observables. Effects beyond the standard collinear factorisation are expected to enhance the radiation of high- $p_{T}$ gluons from the initial state, thus producing an excess of forward jets and/or an excess of multijet events at low $x$ in which the two highest- $E_{T}$ jets are not balanced in transverse momentum. These effects have been investigated in two recent ZEUS analyses: the measurement of forward jet production, extended up to $\eta=4$ thanks to the forward plug calorimeter [11], and the measurement of two- and three-jet events at low $x$ [12]. Figure 7 shows the cross section for two jets that are not back-to-back $(\Delta \Phi<2 \pi / 3)$ for $10<Q^{2}<15 \mathrm{GeV}^{2}$ as a function of $x$ compared to QCD calculations at the order $\alpha_{s}^{2}$ and $\alpha_{s}^{3}$. The highest-order calculation is compatible with the data within a large theoretical uncertainty which reflects the loss of predictability of standard QCD in this region. 

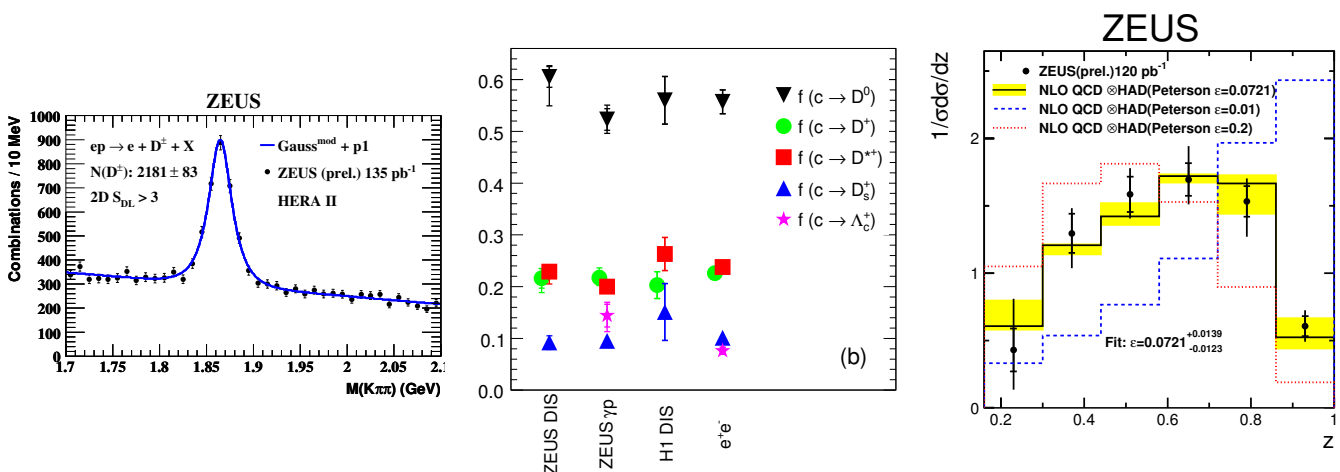

Figure 8: The HERA-II signal for $D^{+}$in DIS (left). Charm fragmentation fractions (centre) and charm fragmentation function (right).

\section{$5 \quad$ Heavy flavours}

Heavy flavour production is an alternative probe of the gluon content of the proton and provides another precise tool to test QCD. New measurements of the production of charmed mesons in DIS are now available, including $D^{*+}, D^{+}, D^{0}, D_{s}^{+}(+$c.c. $) \quad$ from HERA-I data [13] and $D^{*}[14]$ and $D^{+}[15]$ from HERA-II data. The signal-overbackground ratio for the $D^{+} \rightarrow K^{-} \pi^{+} \pi^{+}$ peak at HERA-II largely improved with respect to HERA-I, thanks to the secondary vertexing capability of the silicon microvertex detector. Fig. 8(left) shows the $D^{+}$mass peak after the cut on the lifetime significance in the transverse plane $L_{x y} / \sigma>3$. This improvement makes the $D^{+}$channel competitive to the $D^{*}$ "golden" channel $D^{*+} \rightarrow\left(D^{0} \rightarrow K^{-} \pi^{+}\right) \pi_{S}^{+}$for charm tagging.

To compare the $D$ meson cross sections to the perturbative QCD predictions for charm quarks, some knowledge of the nonperturbative fragmentation of charm quarks into hadrons is needed. The fragmentation fractions of charm into different hadrons have been now directly measured in DIS, as

ZEUS

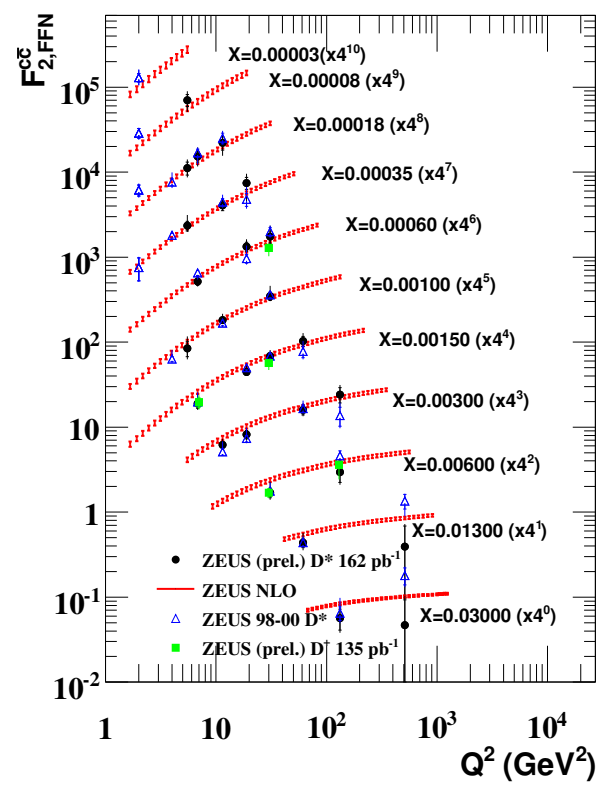

Figure 9: $F_{2}^{c \bar{c}}$ measured from $D^{*+}$ and $D^{+}$, compared with NLO predictions based on the ZEUS PDFs. shown in Fig. 8(center) where they are compared to the previous ZEUS measurement in photoproduction and to $\mathrm{H} 1$ and $e^{+} e^{-}$data. The ZEUS measurements have similar precision to the combined $e^{+} e^{-}$data and support 
the process independence of charm fragmentation [13]. The charm fragmentation function $f(z)$ has been measured in events with a $D^{*+}$ meson associated to a jet [16]. Figure 8(right) shows the measured $f(z)$ compared to NLO predictions implementing different values of the Peterson fragmentation parameter $\epsilon$. Here $z$ is defined as the fraction of the jet $E+P_{\|}$ taken by the $D^{*+}$ meson. A relatively large value of $\epsilon, \epsilon=0.072_{-0.012}^{+0.014}$, gives the best fit within fixed-order NLO theory.

Using the fragmentation parameters and with some extrapolation outside the detector acceptance, the D meson cross sections have been transformed into the charm contribution to the proton structure function, $F_{2}^{c \bar{c}}$. Figure 9 shows $F_{2}^{c \bar{c}}$ as a function of $Q^{2}$ for fixed values of $x$, as obtained from HERA-I and HERA-II $D^{*+}$ and from HERA-II $D^{+}$mesons. The HERA-II data improve the precision of the measurement at large $x$ and $Q^{2}$. The data show clearly a strong scaling violation, in agreement with the QCD predictions based on the ZEUSJETS PDF fit which includes DIS and jet data but not heavy flavour data, thus providing an independent check of the input PDFs.

In principle beauty production could be an even more precise testing ground for QCD since perturbative calculations should be more reliable thanks to the large $b$-quark mass. However, beauty tagging is experimentally more difficult, mainly because of its small cross section. Therfore different experimental techniques have been used. The latest ZEUS measurements exploited $D^{*+} \mu[17]$ or $\mu \mu$ [18] correlations to reduce the background and access the low- $p_{T}^{b}$ region that dominates the total $b \bar{b}$ cross section and was not accessed by previous analyses based on jets.

Figure 10 shows the $b$-quark $p_{T}$ spectrum as reconstructed from different measurements of beauty photoproduction at HERA. The data are in general in agreement with the NLO QCD theory. At low $p_{T}$ the data tend to cluster somewhat above the theoretical uncertainty band. More precise data would be needed to decide whether it is a real deviation from the theory or a fluctuation.

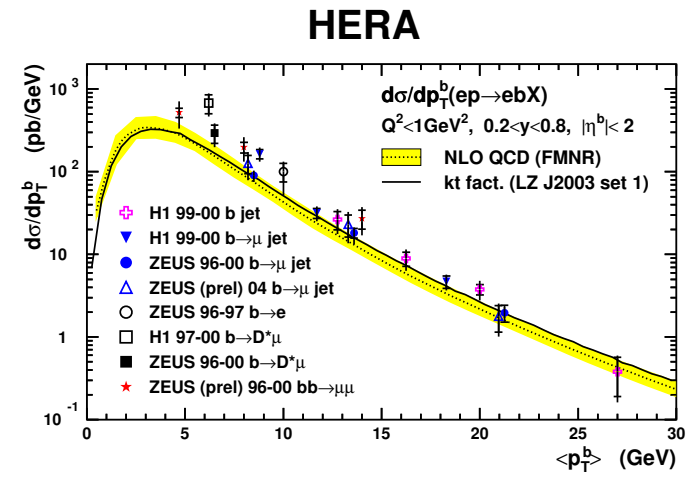

Figure 10: Compilation of ZEUS and H1 measurements of beauty photoproduction shown as a function of the $b$-quark transverse momentum.

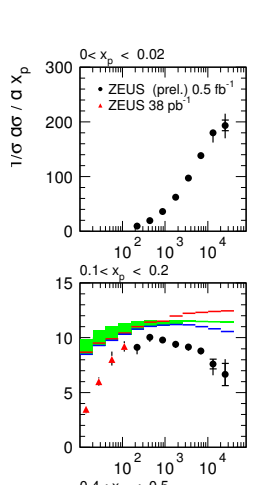

\section{ZEUS}
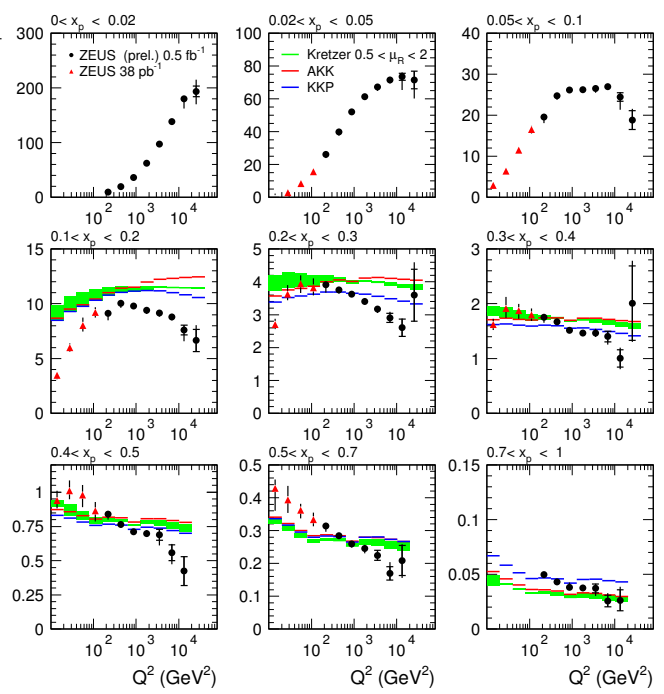

Figure 11: Scaling violation of charged particle fragmentation. 

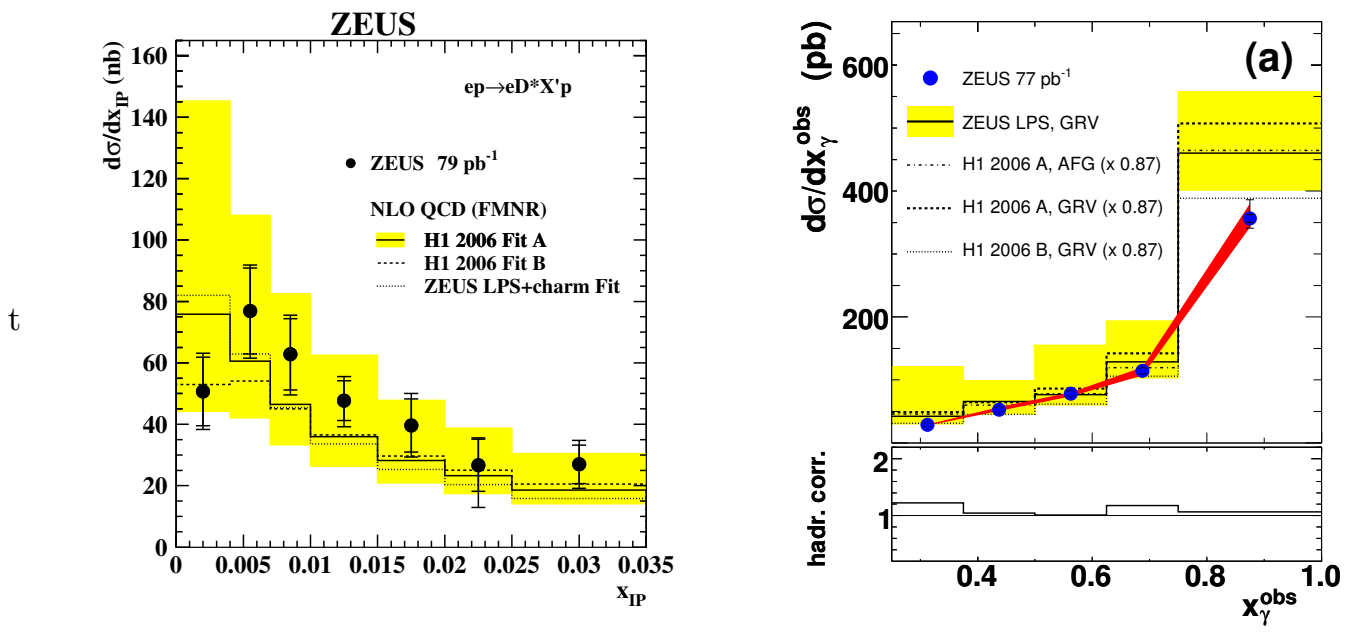

Figure 12: Diffractive $D^{*+}$ (left) and dijet (right) cross sections in photoproduction, compared to NLO QCD predictions using diffractive PDFs

\section{Particle production}

The light-flavour fragmentation functions (FFs), defined as the probability that a final state parton $p$ produces a hadron $h$ with fractional momentum $x_{p}$, are the final-state analogue of the parton densities. Similarly to parton densities, they obey QCD evolution in $Q^{2}$. A measurement of inclusive charged particle fragmentation has been performed in NC DIS, using almost the full HERA data sample, which allowed to extend the previous measurements up to $Q^{2}=40000 \mathrm{GeV}^{2}[19]$. Experimentally $x_{p}$ has been defined in the current hemisphere of the Breit frame as $x_{p}=2 p_{h}^{\text {Breit }} / Q$, where $p_{h}^{\text {Breit }}$ is the hadron momentum. Figure 11 shows the measured charged particles rate $\frac{1}{\sigma} \frac{d \sigma}{d x_{p}}$ in bins of $x_{p}$ as a function of $Q^{2}$. At low $x_{p}$ and low $Q^{2}$ (i.e. where $p_{h} \sim<1 \mathrm{GeV}$ ), particle production is suppressed by hadronisation effects. At large $x_{p}$, a negative logarithmic scaling violation is observed. NLO predictions, based on FFs measured at LEP, are also shown. They reproduce the behaviour of the data qualitatively but predict a milder $Q^{2}$ dependence.

For the new results on the production of heavy stable particle $(p, \bar{p}, d, \bar{d})$ and on BoseEinstein correlations between identified kaons, the reader can refer to the recent publications $[20,21]$.

\section{Diffraction}

Three different characteristics of diffractive events have been used to select diffraction at ZEUS: the presence of a proton carrying a large fraction of the initial proton momentum, which can be tagged by the ZEUS Leading Proton Spectrometer (LPS method); the presence of a large rapidity gap between the hadronic system $X$ observed in the central detector and the forward beam pipe where the proton escapes undetected (LRG method); the small mass of the observed hadronic system $X$ ( $M_{X}$ method). The analysis is in progress and the three 
ZEUS

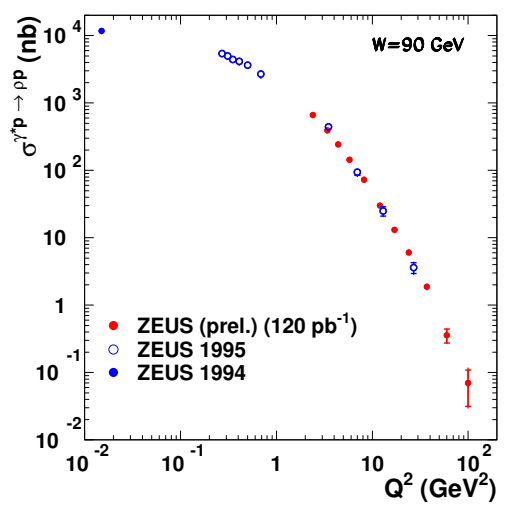

ZEUS

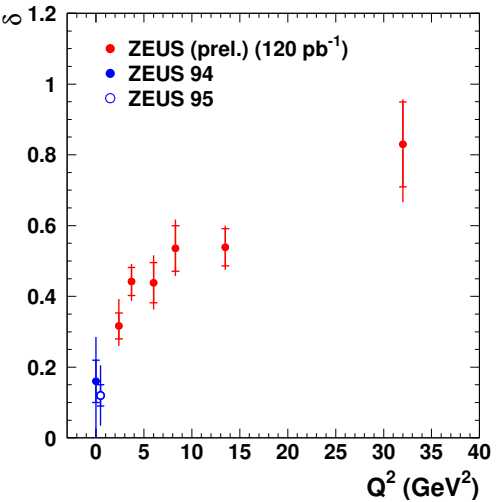

Figure 13: $Q^{2}$ dependence of the exclusive $\rho$ cross section (left). The power $\delta\left(Q^{2}\right)$ from a fit of the form $\sigma(\gamma p \rightarrow \rho p) \propto W^{\delta\left(Q^{2}\right)}$ is shown on the right.

methods are in reasonable agreement, as reported elsewhere in these proceedings [22].

From the QCD factorisation theorem it is expected that diffractive parton density functions (dPDFs), extracted from diffractive DIS cross sections in analogy to the standard PDFs, can be used to compute diffractive cross sections for other (hard) processes. It is well known that this approach fails when applied to dijet production in $p \bar{p}$ collisions [23], where the diffractive cross section is about a factor 10 lower than what is obtained using dPDFs extracted from HERA data. This failure is generally explained as an effect of the rescattering of the proton remnants which destroys the rapidity gap. A similar suppression of diffractive events due to rescattering could be expected also in photoproduction, in particular in resolved-photon events in which the photon behaves similarly to a hadron. The measurement of diffractive photoproduction of $D^{*}$ mesons and of dijets has been finalised recently. Figure 12(left) shows the cross section for diffractive $D^{*}$ production as a function of the fractional energy loss of the proton, $x_{I P}$, compared to NLO QCD predictions based on dPDFs from diffractive DIS data [24]. The agreement is good, supporting the validity of QCD factorisation in diffraction. In the right panel, the dijet cross section for $x_{I P}<0.025$ and for jets with $E_{T}>7.5,6.5 \mathrm{GeV}$ is shown as a function of $x_{\gamma}^{\text {obs }}$ and compared to a preliminary NLO QCD calculation based on dPDFs. The theory agrees with the data and is not compatible with a large suppression in the low- $x_{\gamma}^{\text {obs }}$ region dominated by resolved events. This suggests that indeed photons in high- $E_{T}$ interactions are not behaving exactly like hadrons. Further investigations are ongoing to understand if these results are compatible with a similar analysis recently published by $\mathrm{H} 1$ [26].

The precise measurement of exclusive $\rho^{0}$ production has been extended to large $Q^{2}$, where perturbative QCD calculations are expected to be reliable. The cross section for $\gamma^{*} p \rightarrow \rho^{0} p$ decreases steeply with $Q^{2}$, as shown in Fig. 13, and increases with the $\gamma p$ center-of-mass energy $W$ as $W^{\delta\left(Q^{2}\right)}$. The power $\delta$ increases from values comparable to those found in "soft" hadron-hadron scattering, at $Q^{2} \sim 0$, to values more typical of hard scattering at large $Q^{2}$, in qualitative agreement with QCD predictions [27]. 


\section{ZEUS}
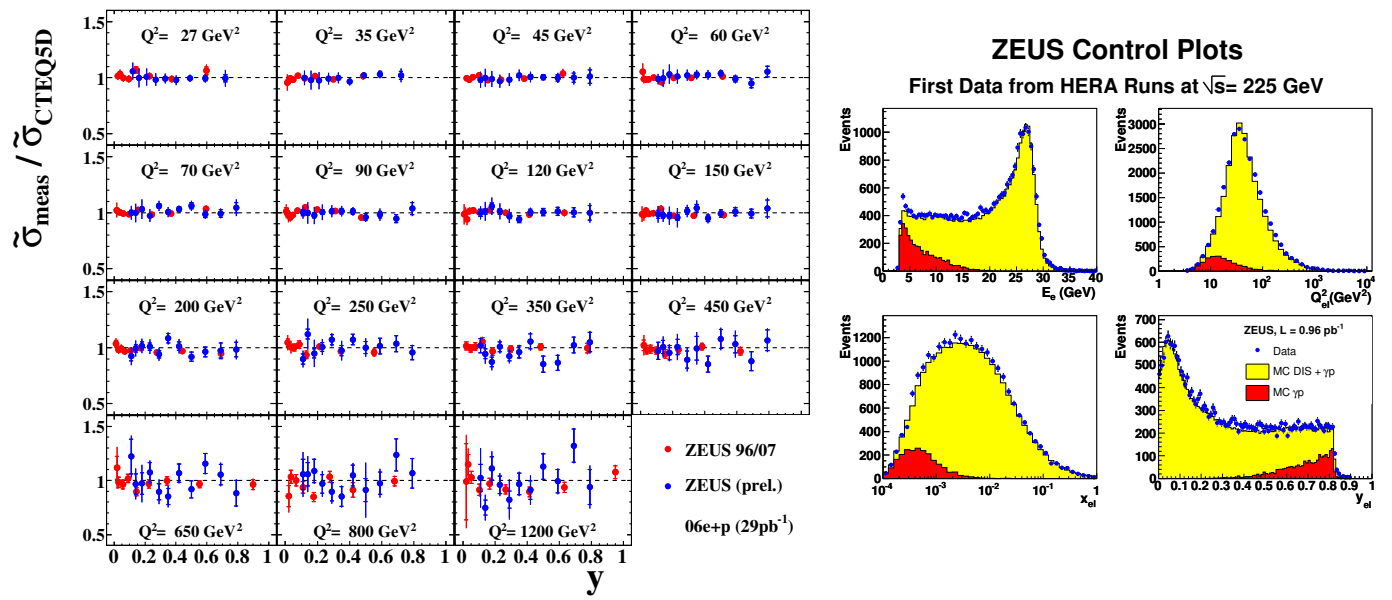

Figure 14: Left: ratio between the measured reduced DIS cross sections and the expectation from CTEQ5D PDFs. The blue points show the new high-y data, the red points are from the standard HERA-I analysis. Right: first data from low energy runs, compared to Monte Carlo

\section{High $y$ and $F_{L}$}

The reduced cross-section at low $Q^{2}$ can be written as

$$
\tilde{\sigma}\left(x, Q^{2}\right)=F_{2}\left(x, Q^{2}\right)-\frac{y^{2}}{Y^{+}} F_{L}\left(x, Q^{2}\right)
$$

To disentangle $F_{L}$ from $F_{2}$, it is therfore needed to vary the factor $\frac{y^{2}}{Y^{+}}$keeping $x$ and $Q^{2}$ fixed. This can be accomplished by varying the $e p$ center of mass energy, since $y=Q^{2} /(x s)$, which is the main reason for the current runs with reduced proton energy. To maximise the sensitivity to $F_{L}$, the DIS measurement has to be extended to the highest possible $y$. High- $y$ corresponds to low scattered-electron energy and therefore the largest accessible $y$ is limited by the ability to reconstruct and identify low-energy electrons keeping the background under control.

A first extension of the ZEUS DIS kinematic coverage towards higher $y$ has been attempted using $29 \mathrm{pb}^{-1}$ of data taken in 2006 in high-energy runs with a special trigger for low-energy electrons, similar to that being used in the low-energy running. The results are presented as a ratio to the theory (CTEQ5D) and compared to previous ZEUS data in Fig. 14(left). The improvement at large $y$ and low $Q^{2}$ is apparent [28].

The HERA running at reduced beam energy has been proceeding smoothly. At the time of this conference ZEUS collected about $3 \mathrm{pb}^{-1}$. The distribution of the electron energy, $Q^{2}, x$ and $y$ from these data are shown in Fig. 14(right) where they are compared to Monte Carlo distributions for DIS and for the photoproduction background. The good agreement shows the level of understanding of these low-energy data. 


\section{Postscript}

At the time of writing (July 2007), HERA has been switched off and the dismantling of the ZEUS detector has already started. The low energy running has been more succesfull than expected, with $13 \mathrm{pb}^{-1}$ collected at $\sqrt{s}=225 \mathrm{GeV}$ and $7 \mathrm{pb}^{-1}$ at an intermediate energy point at $\sqrt{s}=252 \mathrm{GeV}$. Obviously this is not the end of ZEUS, since the final and most precise physics results, based on the full luminosity and on the low-energy data, are still to come.

\section{References}

[1] Slides: http://indico. cern. ch/contributionDisplay $\cdot$ py? contribId=3\&sessionId=2\&conf Id=9499

[2] S. Bhadra, these proceedings.

[3] H1 and ZEUS collaborations, contributed paper to the ICHEP06 conference, http://www-h1.desy.de/h1/www/publications/htmlsplit/H1prelim-06-142.long.html.

[4] ZEUS collaboration, contributed paper to ICHEP06 http://www-zeus.desy.de/physics/phch/conf/ichep06/hiq2/8/ZEUS-prel-06-018. pdf.

[5] O. Ota, these proceedings.

[6] K. Korcsak-Gorzo, these proceedings.

[7] ZEUS Collaboration; S. Chekanov et al., Phys. Lett. B 64912 (2007); ZEUS Collaboration; S. Chekanov et al.,Nucl. Phys. B 7651 (2007).

[8] T. Schoerner-Sadenius, these proceedings.

[9] H. Wolfe, these proceedings.

[10] ZEUS Collaboration, S. Chekanov et al., DESY-07-092, arXiv:0706.3809 [hep-ex], submitted to Phys. Rev. D.

[11] ZEUS Collaboration, S. Chekanov et al., DESY-07-100, submitted to Eur. Phys. J. C.

[12] ZEUS Collaboration, S. Chekanov et al., DESY-07-062, arXiv:0705.1931 [hep-ex], published online by Nuc. Phys. B.

[13] ZEUS Collaboration, S. Chekanov et al., DESY-07-052, arXiv:0704.3562 [hep-ex], accepted by JHEP.

[14] H. Stadie, these proceedings.

[15] D. Nicholass, these proceedings.

[16] S. Fang, these proceedings.

[17] ZEUS collaboration, S. Chekanov et al., Eur. Phys. J. C 50299 (2007).

[18] ZEUS collaboration, contributed paper to ICHEP06, http://www-zeus.desy.de/physics/phch/conf/ichep06/hfl/6/ZEUS-prel-05-008-updated.ps

[19] B. Brzozowska, these proceedings.

[20] ZEUS Collaboration; S. Chekanov et al. DESY-07-070, arXiv:0705.3770 [hep-ex], published online in Nucl. Phys. B.

[21] ZEUS Collaboration; S. Chekanov et al., DESY-07-069, arXiv:0706.2538 [hep-ex], published online in Phys. Lett.B.

[22] J. Lukasik, these proceedings; B. Loehr, these proceedings.

[23] CDF Collaboration, T. Affolder et al., Phys. Rev. Lett. 84, 5043 (2000).

[24] ZEUS Collaboration; S. Chekanov et al., Eur.Phys.J. C 51301 (2007).

[25] Y. Yamazaki, these proceedings.

[26] H1 Collaboration, A. Aktas et al., DESY-07-018, hep-ex/0703022, submitted to Eur. Phys. J.C.

[27] A. Levy, these proceedings.

[28] S. Shimizu, these proceedings. 